\title{
Endoscopic Resection of Ampullary Neuroendocrine Tumor
}

\author{
Hiroyuki Fukasawa, Shigetaka Tounou, Masashi Nabetani and Tomoki Michida
}

\begin{abstract}
We report the case of a 57-year-old man with a 1.0-cm grade 1 neuroendocrine tumor (G1 NET) of the ampulla of Vater (ampullary NET) who underwent endoscopic snare papillectomy. Pancreatitis occurred after endoscopic resection but was cured with conservative therapy. In two years of follow-up, no local recurrence or metastasis occurred. Endoscopic snare papillectomy for small G1 NET of the ampulla of Vater is relatively safe and less invasive than surgical treatment and should be considered as treatment option.
\end{abstract}

Key words: ampullary neuroendocrine tumor, endoscopic papillectomy

(Intern Med 56: 499-503, 2017)

(DOI: 10.2169/internalmedicine.56.7520)

\section{Introduction}

Neuroendocrine tumor (NET) of the ampulla of Vater (ampullary NET) is very rare, and a standard therapy for small, low-grade NET $(\leq 1.0 \mathrm{~cm})$ has not been established (1-4). Surgical resection by such procedures as pancreaticoduodenectomy or local resection has been recommended, but pancreaticoduodenectomy is a relatively invasive surgery. While there have been studies on endoscopic resection recently (5-9) and the prognosis of low-grade NET is known to be good, it has yet to be established as a treatment option for small, low-grade ampullary NET. When small ampullary NET is diagnosed as being located within the submucosal layer and there is no evidence of metastasis with contrast medium-enhanced multi-detector computed tomography (MDCT) and endoscopic ultrasonography (EUS), endoscopic therapy should be considered, in light of its minimal invasiveness. We herein report the successful endoscopic snare papillectomy of a small, grade 1 neuroendocrine tumor of the ampulla of Vater.

\section{Case Report}

A 57-year-old asymptomatic man had undergone esophagogastroduodenoscopy (EGD) annually in a medical check-up for the past 3 years. A small, protruding lesion had been observed on the anal side of the ampulla of Vater since the first check-up (Fig. 1), but no endoscopic biopsy or a more detailed examination had been performed because the lesion was small and asymptomatic. The protruding lesion was enlarged in the latest EGD (Fig. 2), and endoscopic biopsy was conducted. The histological diagnosis was lowgrade neuroendocrine tumor, and a more detailed examinations with enhanced MDCT, EUS, and hypotonic duodenography was performed. No distant metastasis or lymph node metastasis was found with MDCT (Fig. 3). MDCT also revealed a smooth pancreatic duct just beneath the ampulla of Vater (Fig. 4), and we decided not to perform magnetic resonance cholangiopancreatography because it was not considered to be able to provide more information about the pancreatic duct. A $1.0 \times 0.6 \mathrm{~cm}$ iso-echoic homogenous tumor was found to be located within the submucosal layer with EUS (Fig. 5). A smooth-surfaced protruding lesion was observed on the anal side of the ampulla of Vater in hypotonic duodenography (Fig. 6). The risk of pancreatic duct invasion was thought to be low because the lesion was located on the anal side of the ampulla of Vater, apart from the biliary and pancreatic duct orifice, and neither ERCP nor IDUS had been performed to prevent pancreatitis after ERCP.

When we explained the findings of the detailed examinations to the patient and his family and discussed the treatment of the tumor with them, they desired endoscopic resection rather than surgical resection, and thus endoscopic snare papillectomy was performed. Bleeding from the resection site occurred immediately after resection, and endoscopic hemostasis was performed with soft coagulation using bipolar hemostatic forceps (H-S2518 Pentax Medical). A plastic 


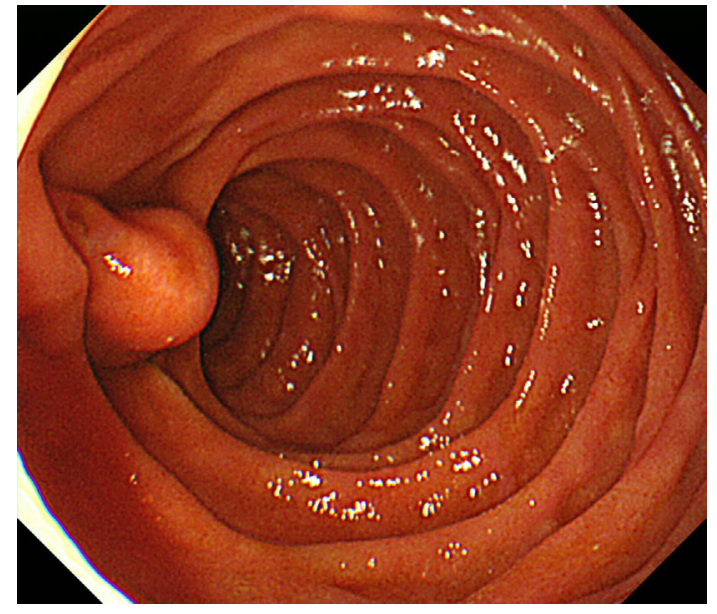

Figure 1. Endoscopic image of the ampulla of Vater performed three years prior. A small protrusion was found on the anal side of the ampulla of Vater.



Figure 3. Multi-detector computed tomography view. A small tumor $10 \mathrm{~mm}$ in diameter was located in the second portion of duodenum and was weakly enhanced with contrast medium (arrowhead).

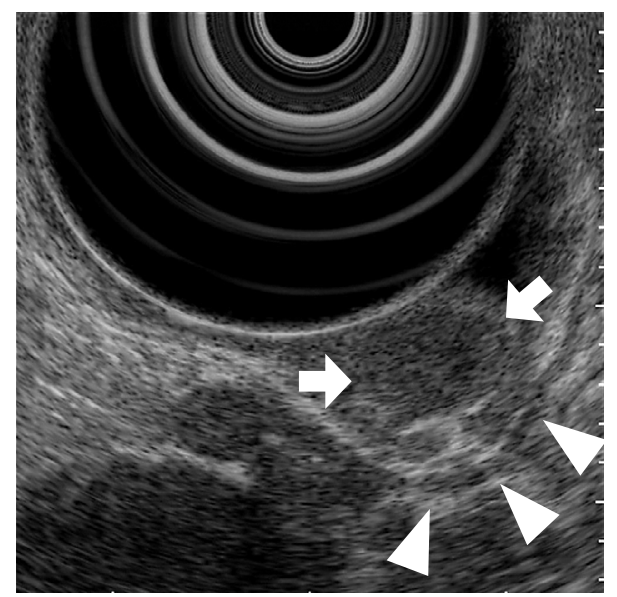

Figure 5. Endoscopic ultrasonography image. A tumor (arrows) was located within the mucosa or submucosal layer, apart from the muscularis propria (arrowheads).



Figure 2. Endoscopic image of the ampulla of Vater performed three months before endoscopic therapy. The protrusion was larger than three years prior.

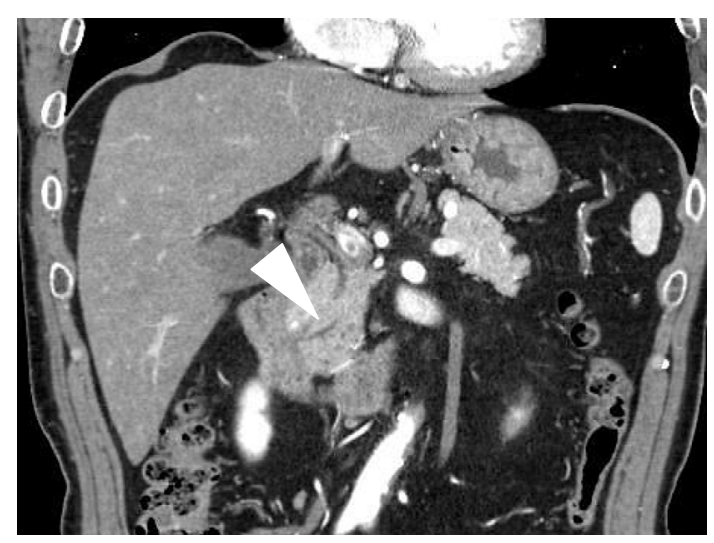

Figure 4. Multi-detector computed tomography coronal view. A smooth pancreatic duct just beneath the ampulla of Vater was clearly found (arrowhead).

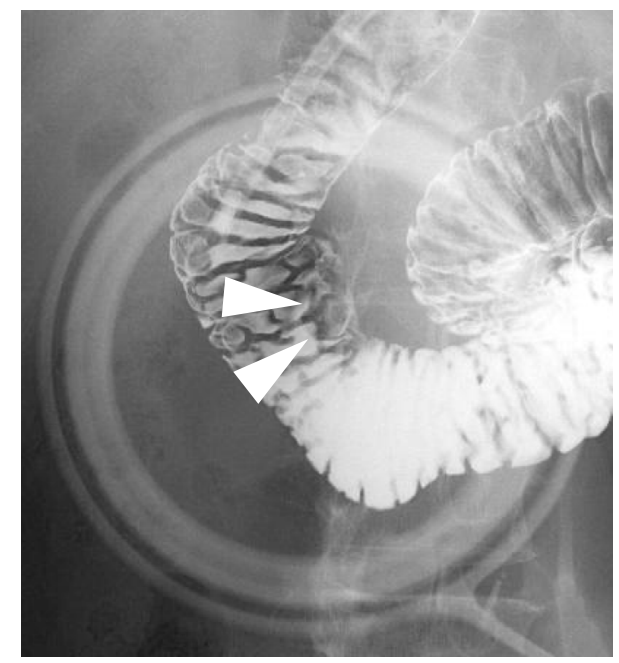

Figure 6. A smooth-surfaced protruding lesion was found on the anal side of the ampulla of Vater in hypotonic duodenography (arrowheads). 


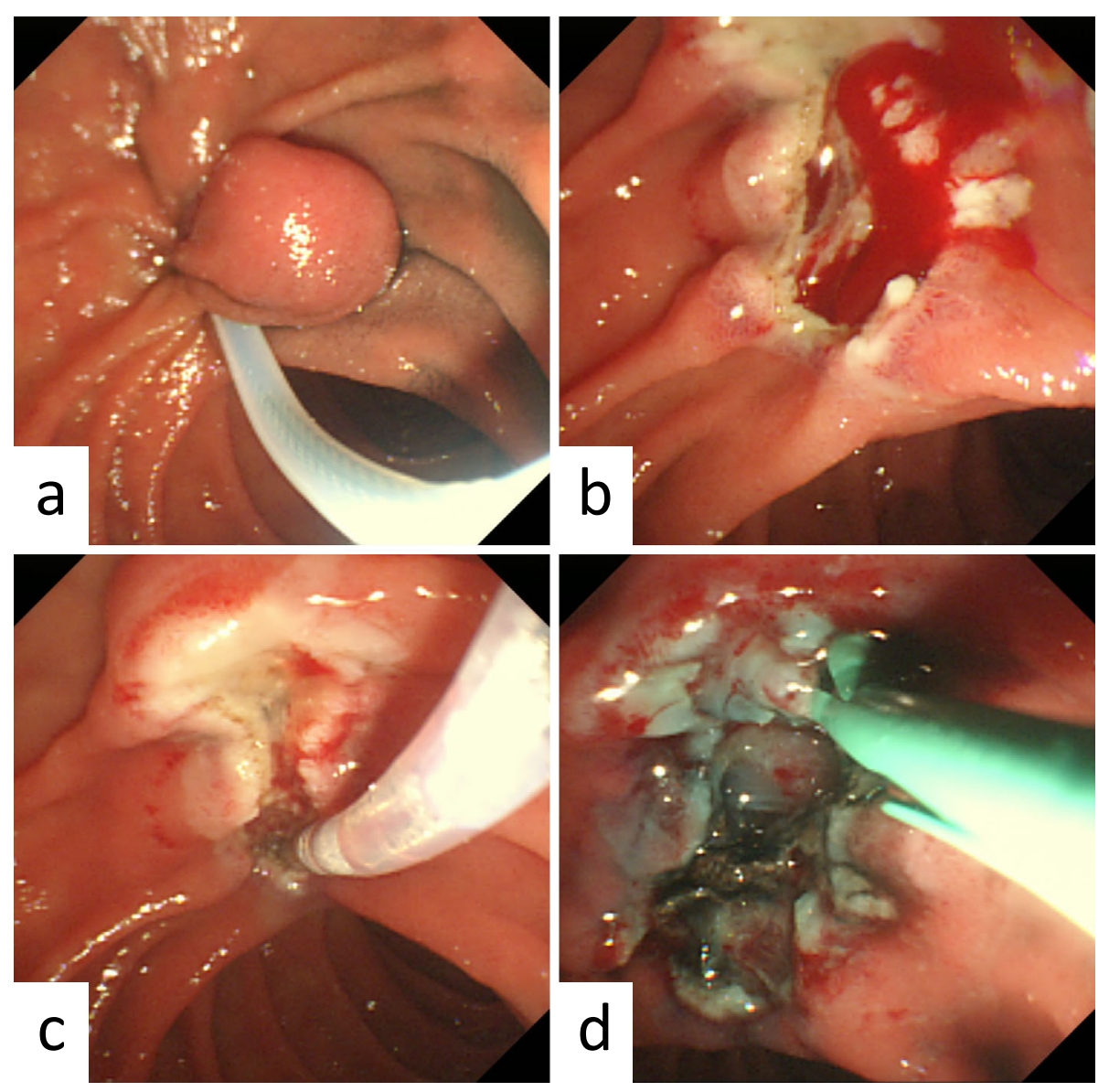

Figure 7. Endoscopic papillectomy for G1 NET of the ampulla of Vater. (a) Endoscopic snare papillectomy. (b) Immediate bleeding after papillectomy. (c) Endoscopic hemostasis using bipolar hemostatic forceps. (d) A plastic stent was placed in the pancreatic duct, but a biliary stent could not be placed.

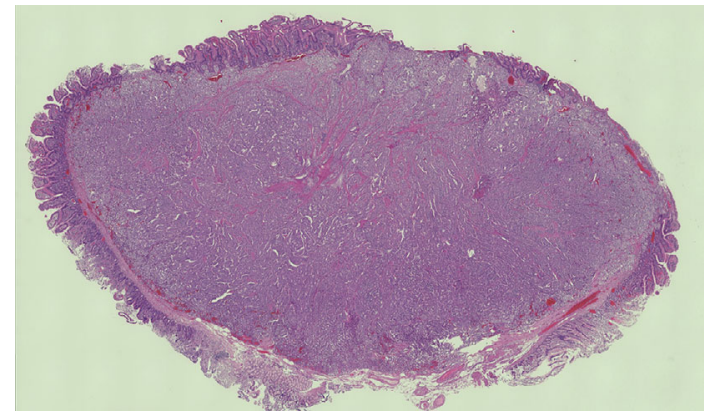

Figure 8. Low-power $(\times 10)$ histological view of the resected specimen. The tumor size was $1.0 \times 0.6 \mathrm{~cm}$ in diameter.

stent was placed into the pancreatic duct to prevent pancreatitis, but as we could not identify the opening of the bile duct, a biliary stent for the common bile duct could not be placed (Fig. 7). After endoscopic resection, acute pancreatitis occurred following the spontaneous drop-out of the pancreatic stent, but this was cured with conservative treatment.

The histological diagnosis of the resection specimen was $1.0 \times 0.6 \mathrm{~cm}$ low grade (G1) NET (Fig. 8). Immunochemical histology showed positive synaptophysin staining, positive CD56 staining, positive chromogranin A staining, and a Ki67 index of $<2 \%$ (Fig. 9). The neuroendocrine tumor was located within the submucosal layer, and neither muscularis propria invasion nor lymph vascular invasion was found. Additional treatment was not planned, due to the absence of metastasis and poor prognostic properties.

\section{Discussion}

The incidence of low-grade NET is estimated to be 1.62.0 cases per 100,000 persons per year, and ampullary NET only accounts for $0.05 \%$ of low-grade NETs (3). Endoscopic resection is considered to be a treatment choice for small $(\leq 1.0 \mathrm{~cm})$ low-grade NET of the gastrointestinal tract, but it is still uncertain whether it is an option for the treatment of small, low-grade ampullary NET (4). Surgical resection, such as by pancreaticoduodenectomy, is the most reliable form of treatment, but surgical resection is invasive. Generally, for an ampullary NET, a large size of more than 2.0 $\mathrm{cm}$ is considered an indication for surgical resection. Successful endoscopic resection has been reported for some ampullary NETs (5-9) as well as for some relatively large-sized NETs. Yi et al. reported successful endoscopic submucosal resection for an ampullary NET $2.5 \mathrm{~cm}$ in diameter, but the follow-up was only 6 months, and the long-term prognosis was unknown (8). Odabasi et al. reported successful endo- 

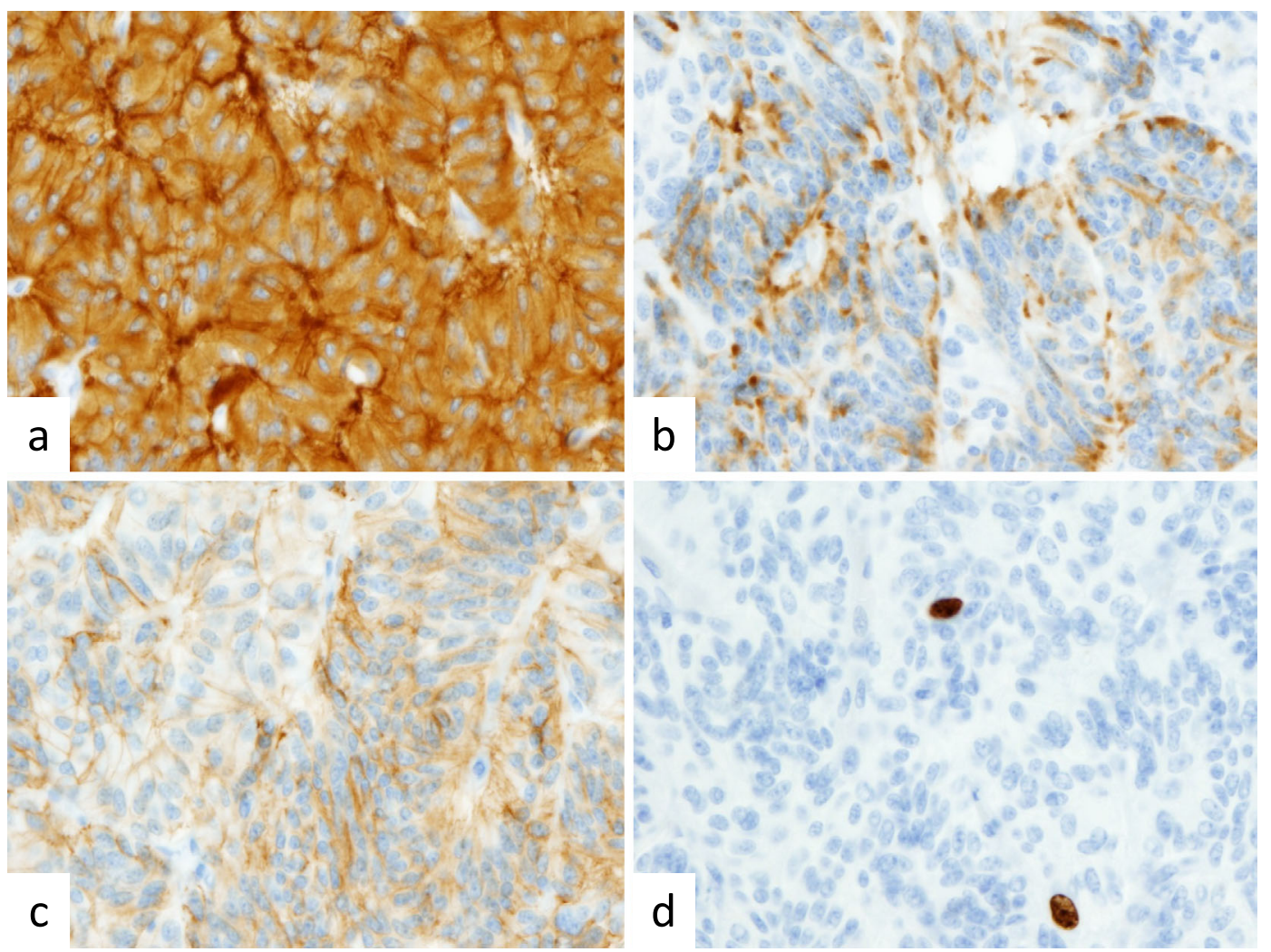

Figure 9. Immunochemical histology of the resected specimen $(\times 200)$. (a) Positive synaptophysin staining. (b) Positive CD56 staining. (c) Positive chromogranin A staining. (d) The Ki-67 index was less than $2 \%$.

scopic snare papillectomy for an ampullary NET $1.9 \mathrm{~cm}$ in diameter with no recurrence or distant metastasis in 14 months of follow-up (9). Two cases of successful endoscopic resection for ampullary NETs $1.5 \mathrm{~cm}$ in diameter $(6,7)$ have also been reported. However, given that small ampullary NETs $(1.2 \mathrm{~cm}$ in diameter and $0.7 \mathrm{~cm}$ in diameter) with lymph node metastasis have also been reported $(10,11)$, long-term follow-up is necessary after endoscopic resection of ampullary NETs.

In the present case, the submucosal tumor was diagnosed as a low-grade NET through a histological diagnosis of a forceps biopsy specimen. It was located within the submucosal layer and diagnosed as small in size $(\leq 1.0 \mathrm{~cm})$ by EUS before endoscopic resection. No lymph node metastasis or distal metastasis was found with contrast mediumenhanced MDCT before the treatment. No lymphovascular invasion was found in the histological diagnosis of the resected specimen, and no local recurrence or distant metastasis was observed in contrast medium-enhanced MDCT or endoscopy for two years after endoscopic resection. In conclusion, the ideal treatment for small, low-grade, ampullary NET is still unknown, but endoscopic resection and followup without surgical resection is considered a treatment option when the size of the NET is smaller than $1 \mathrm{~cm}$ (for low-grade NET of the gastrointestinal tract), there is no evidence of lymph node metastasis or invasion of muscularis propria in EUS and enhanced MDCT, and a resected specimen reveals no sign of lymphovascular invasion.
Low-grade NET of the ampulla of Vater is considered to have a good prognosis, but lymph node metastasis or distal metastasis may occur a long time after resection, so longterm detailed follow-up should be performed, even if there is no sign of lymphovascular invasion.

The authors state that they have no Conflict of Interest (COI).

\section{References}

1. Ricci JL. Carcinoid of the ampulla of Vater: local resection or pancreaticoduodenectomy. Cancer 71: 686-690, 1993.

2. Hatzitheoklitos E, Büchler MW, Friess H, et al. Carcinoid of the ampulla of Vater. Clinical characteristics and morphologic features. Cancer 73: 1580-1588, 1994.

3. Hartel M, Wente MN, Sido B, Friess H, Büchler MW. Carcinoid of the ampulla of Vater. J Gastroenterol Hepatol 20: 676-681, 2005.

4. Nikou GC, Toubanakis C, Moulakakis KG, et al. Carcinoid tumors of the duodenum and the ampulla of Vater: current diagnostic and therapeutic approach in a series of 8 patients. Case series. Int $\mathbf{J}$ Surg 9: 248-253, 2011.

5. Pyun DK, Moon G, Han J, et al. A carcinoid tumor of the ampulla of Vater treated by endoscopic snare papillectomy. Korean J Intern Med 19: 257-260, 2004.

6. Gilani N, Ramirez FC. Endoscopic resection of an ampullary carcinoid presenting with upper gastrointestinal bleeding: a case report and review of the literature. World J Gastroenterol 13: 12681270, 2007.

7. Fukatsu H, Kawamoto H, Fujii M, et al. Periampullary carcinoid tumor. Endoscopy 39 Suppl 1: E49-E50, 2007. 
8. Yi H, Wu C, Mou Y, et al. Successful en bloc resection of papillary neuroendocrine tumors by duodenoscope using endoscopic submucosal dissection method. Clin Res Hepatol Gastroenterol 36: e100-e103, 2012.

9. Odabasi M, Yildiz KM, Cengiz E, et al. Treatment of ampullary neuroendocrine tumor by endoscopic snare papillectomy. Am J Case Rep 14: 439-443, 2013.

10. Matsubayashi H, Matsunaga K, Sasaki K, Yamaguchi Y, Hasuike N, Ono H. Small carcinoid tumor of papilla of the Vater with lymph node metastases. J Gastrointest Cancer 39: 61-65, 2008.

11. Senda E, Fujimoto K, Ohnishi K, et al. Minute ampullary carcinoid tumor with lymph node metastases: a case report and review of literature. World J Surg Oncol 7: 9, 2009.

The Internal Medicine is an Open Access article distributed under the Creative Commons Attribution-NonCommercial-NoDerivatives 4.0 International License. To view the details of this license, please visit (https://creativecommons.org/licenses/ by-nc-nd/4.0/).

(C) 2017 The Japanese Society of Internal Medicine http://www.naika.or.jp/imonline/index.html 